\title{
Induction of Apoptosis in Human Oral Keratinocyte by Doxorubicin
}

\author{
HIROSHI SAKAGAMI ${ }^{1,2}$, NORIYUKI OKUDAIRA ${ }^{1}$, YOSHIKO MASUDA ${ }^{2}$, \\ OSAMU AMANO ${ }^{3}$, SATOSHI YOKOSE ${ }^{4}$, YUMIKO KANDA $^{5}$, MADOKA SUGURO ${ }^{6}$, \\ TAKENORI NATORI ${ }^{6}$, HIROSHI OIZUMI $^{6}$ and TAKAAKI OIZUMI ${ }^{6}$ \\ ${ }^{1}$ Division of Pharmacology, Meikai University School of Dentistry, Sakado, Japan; \\ ${ }^{2}$ Meikai Pharmaco-Medical Laboratory (MPL), Meikai University School of Dentistry, Sakado, Japan; \\ ${ }^{3}$ Division of Anatomy, Meikai University School of Dentistry, Sakado, Japan; \\ ${ }^{4}$ Division of Endodontics, Meikai University School of Dentistry, Sakado, Japan; \\ ${ }^{5}$ Department of Electron Microscope, Meikai University, School of Dentistry, Sakado, Japan; \\ ${ }^{6}$ Daiwa Biological Research Institute Co, Ltd., Kanagawa, Japan
}

\begin{abstract}
Background/Aim: We have previously reported that doxorubicin (DXR) showed much higher cytotoxicity against human oral squamous cell carcinoma cell lines compared to normal human mesenchymal normal oral cells (gingival fibroblast, periodontal ligament fibroblast, pulp cell), yielding high tumor-specificity. However, we unexpectedly found that doxorubicin showed potent cytotoxicity against human normal oral keratinocytes and primary gingival epithelial cells. In the present study, we investigated the reproducibility, underlining mechanisms and generality of this unexpected finding. Materials and Methods: Viable cell number was determined by the 3-14,5dimethylthiazol-2-yl)-2,5-diphenyltetrazolium bromide method, fine cell structure by transmission electron microscopy and apoptosis induction by western blot analysis. Results: Doxorubicin induced keratinocyte toxicity, regardless of cell density and concentration of FBS in the culture medium. Doxorubicin induced apoptosis (characterized by the loss of cell surface microvilli, chromatin condensation, nuclear fragmentation and caspase-3 activation) in keratinocytes. A total of 11 anticancer drugs showed similar keratinocyte toxicity. Alkaline extract of the leaves of Sasa senanensis Rehder partially alleviated the DXR-induced keratinocyte cytotoxicity by
\end{abstract}

This article is freely accessible online.

Correspondence to: Hiroshi Sakagami, Division of Pharmacology, Department of Diagnostic and Therapeutic Sciences, Meikai University School of Dentistry, Sakado, Saitama 350-0283, Japan. Tel: +81 492792758, Fax: +81 492855171, e-mail: sakagami@dent.meikai.ac.jp; takaakio@daiwaseibutsu.co.jp

Key Words: Cytotoxicity, tumor selectivity, oral carcinoma, mesenchymal and epithelial normal oral cells, keratinocyte toxicity. promoting cell growth. Conclusion: The present study suggested that oral keratinocyte toxicity is a novel adverse effect of most anticancer agents.

For the exploration of new anticancer drugs, experiments with tumor-bearing animals are mandatory. However, the results obtained with animals have to be carefully evaluated, since drug metabolisms are quite different between animals and humans $(1,2)$. The other approach is to use cell cultures that mimic whole body. We have established an in vitro assay method of anti-tumor activity, based on the relative cytotoxicity against human oral squamous cell carcinoma (OSCC) cell lines (Ca9-22, HSC-2, HSC-3, HSC-4) and human normal oral mesenchymal cells (gingival fibroblast, HGF; periodontal ligament fibroblast, HPLF; pulp cell, HPC) (3). The validity of this model for estimating the antitumor activity was obtained by our finding that anticancer drugs such as topoisomerase I inhibitors (camptothecin, SN-38) and topoisomerase II inhibitors (etoposide, teniposide), anthracyclines (doxorubicin, daunorubicin, epirubicin, mitoxantrone), mitomycin $\mathrm{C}$, docetaxel, 5-FU and bacterial products (nocobactin NA-a, -b) showed one or two-orders higher tumor-specificity than hundreds of other natural products including tannins, flavonoids and other lower molecular weight polyphenols (3).

We recently found that doxorubicin showed very potent cytotoxicity against human normal oral epithelial cells (mucosa keratinocyte, HOK; primary gingival epithelial cell, HGEP) (4-6). However, the mechanism of keratinocyte toxicity is obscure. In the present study, we investigated whether keratinocyte toxicity is reproducibly observable, regardless the culturing conditions and types of anticancer drugs, and whether it is coupled with apoptosis induction. We also present our preliminary data that searched natural products for their ability to alleviate the keratinocyte toxicity. 
Table I. Cytotoxic activity of anticancer drugs against human oral malignant and non-malignant cells.

\begin{tabular}{|c|c|c|c|c|c|c|c|c|c|c|c|c|c|c|}
\hline & \multicolumn{12}{|c|}{$\mathrm{CC}_{50}(\mu \mathrm{M})$} & \multicolumn{2}{|c|}{ TS } \\
\hline & & & & & & & & Huma & oral no & rmal cells & & & \multirow{3}{*}{$\begin{array}{c}\text { Mes.nor } \\
v s . \\
\text { OSCC } \\
(\mathrm{B} / \mathrm{A})\end{array}$} & \multirow{3}{*}{$\begin{array}{c}\text { Epi. Nor } \\
v s . \\
\text { OSCC } \\
\text { (C/A) }\end{array}$} \\
\hline & \multicolumn{5}{|c|}{ Human oral squamous cell carcinoma } & \multicolumn{4}{|c|}{ Mesenchumal cells } & \multicolumn{3}{|c|}{ Epithelial cells } & & \\
\hline & $\mathrm{Ca} 9-22$ & HSC-2 & HSC-3 & HSC-4 & $\begin{array}{c}\text { mean } \\
(\mathrm{A})\end{array}$ & HGF & HPLF & HPC & $\begin{array}{l}\text { mean } \\
\text { (B) }\end{array}$ & HOK & HGEP & $\begin{array}{c}\text { mean } \\
(\mathrm{C})\end{array}$ & & \\
\hline CPT & $<0.064$ & $<0.064$ & $<0.064$ & $<0.064$ & $<0.064$ & 200.0 & 9.8 & 146.0 & 118.6 & 0.25 & 3.93 & 2.09 & $>1853$ & $>33$ \\
\hline SN-38 & $<0.064$ & $<0.064$ & $<0.064$ & $<0.064$ & $<0.064$ & 143.0 & 29.3 & 15.7 & 62.7 & $<0.075$ & 1.47 & $<0.77$ & $>979$ & $<12$ \\
\hline DXR & 0.13 & 0.06 & 0.09 & 0.06 & 0.09 & 7.3 & 1.3 & 9.3 & 6.0 & 0.13 & 0.17 & 0.15 & 70 & 1.7 \\
\hline DNR & 0.27 & 0.07 & 0.13 & 0.09 & 0.14 & 4.9 & 10.0 & 8.2 & 7.7 & $<0.0039$ & 0.41 & $<0.21$ & 55 & $<1.5$ \\
\hline ETP & 11.27 & 3.00 & 2.67 & 2.47 & 4.85 & 351.3 & 500.0 & 500.0 & 450.4 & 1.80 & 3.17 & 2.48 & 93 & 0.5 \\
\hline MMC & 3.97 & 0.36 & 0.14 & 0.78 & 1.31 & 22.0 & 65.0 & 34.3 & 40.4 & 0.10 & 0.28 & 0.19 & 31 & 0.1 \\
\hline MTX & 8.97 & 0.19 & $<0.13$ & $<0.13$ & $<2.35$ & $>400$ & $>400$ & $>400$ & $>400$ & $>1000$ & $<0.13$ & $>500$ & $>170$ & $>212$ \\
\hline 5-FU & 15.27 & 100.33 & 186.33 & 92.67 & 98.65 & $>1000$ & $>1000$ & $>1000$ & $>1000$ & 12 & 14 & 13 & $>10$ & 0.1 \\
\hline DOC & $<0.032$ & $<0.032$ & $<0.032$ & $<0.032$ & $<0.032$ & 69.7 & 99.7 & 90.7 & 86.7 & 0.12 & 0.032 & 0.076 & $>2708$ & $>2.4$ \\
\hline Melphalan & 114.00 & 29.00 & 18.33 & 19.00 & 45.08 & 153.3 & 197.3 & 169.7 & 173.4 & 13,47 & 18.67 & 16,07 & 4 & 0.4 \\
\hline Gefitinib & 18.00 & 22.33 & 15.67 & 13.67 & 17.42 & 57.7 & 68.3 & 83.0 & 69.7 & 3.47 & 4.10 & 3.78 & 4 & 0.2 \\
\hline
\end{tabular}

Each value represents the mean of triplicate determinations. HGF, Human gingival fibroblast; HPC, human pulp cells; HPLF, human periodontal ligament fibroblast; Ca9-22 (derived from gingival tissue), HSC-2, HSC-3 and HSC-4 (derived from tongue), human oral squamous cell carcinoma cell lines; $\mathrm{CC}_{50}, 50 \%$ cytotoxic concentration; CPT, camptothecin; DXR, doxorubicin; DNR, daunorubicin; ETP, etoposide; MMC, mitomycin C, 5-FU, 5-fluorouracil; DOC, docetaxel.

\section{Materials and Methods}

Materials. The following chemicals and reagents were obtained from the indicated companies: Dulbecco's modified Eagle's medium (DMEM) from GIBCO BRL, Grand Island, NY, USA; fetal bovine serum (FBS), 3-(4,5-dimethylthiazol-2-yl)-2,5-diphenyltetrazolium bromide (MTT), doxorubicin, daunorubicin, camptothecin, SN-38, etoposide and melphalan from Sigma-Aldrich Inc., St. Louis, MO, USA; mitomycin C from Merck KGaA, Darmstadt, Germany; docetaxel from Toronto Research Chemicals, New York, NY, USA; gefitinib from LC Laboratories ${ }^{\circledR}$, PKC Pharmaceuticals, Inc., Woburn, MA, USA; dimethyl sulfoxide (DMSO) from Wako Pure Chem. Ind., Osaka, Japan; 5-fluorouracil (5-FU) from Kyowa, Tokyo, Japan; methotrexate from Nacalai Tesque, Inc., Kyoto, Japan. Culture plastic dishes and plates (96-well) were purchased from Becton Dickinson (Franklin Lakes, NJ, USA). Alkaline extract of the leaves of Sasa senanensis Rehder (commercial name, Sasahealth) was provided by Daiwa Biological Research Institute Co., Ltd., Kanagawa, Japan.

Cell culture. HGF, HPLF and HPC cells, established from the first premolar tooth extracted from the lower jaw of a 12-year-old girl (7), and OSCC cell lines [Ca9-22 (derived from gingival tissue; HSC-2, HSC-3, HSC-4 (derived from tongue)], purchased from Riken Cell Bank, Tsukuba, Japan, were cultured at $37^{\circ} \mathrm{C}$ in DMEM supplemented with $10 \%$ heat-inactivated FBS, 100 units $/ \mathrm{ml}$ penicillin $\mathrm{G}$ and $100 \mu \mathrm{g} / \mathrm{ml}$ streptomycin sulfate under a humidified $5 \% \mathrm{CO}_{2}$ atmosphere. HOK cells (purchased from COSMO BIO Co/ Ltd., Tokyo, Japan) were cultured in keratinocyte growth supplement (OKGS, Cat, No. 2652). HGEP cells (purchased from
CELLnTEC Advanced Cell Systems AG, Bern, Switzerland) were grown in CnT-PR medium (4-6). HOK and HGEP at 7 11 population doubling levels were used in the present study.

Assay for cytotoxic activity. Cells were inoculated at $2.5 \times 10^{3}$ cells $/ 0.1$ $\mathrm{ml}$ in a 96-microwell plate (Becton Dickinson Labware). After $48 \mathrm{~h}$, the medium was replaced with $0.1 \mathrm{ml}$ of fresh medium containing different concentrations of single test compound. Cells were incubated further for $48 \mathrm{~h}$ and the relative viable cell number was then determined by the MTT method $(5,6)$. The relative viable cell number was determined by the absorbance of the cell lysate at 540 or $562 \mathrm{~nm}$, using a microplate reader (Biochromatic Labsystem, Helsinki, Finland; Sunrise Rainbow RC-R; TECAN, Männedorf, Switzerland, respectively). Control cells were treated with the same amounts of DMSO and the cell damage induced by DMSO was subtracted. $\mathrm{CC}_{50}$ was determined from the dose-response curve and the mean value of $\mathrm{CC}_{50}$ for each cell type was calculated from triplicate assays.

Western blot analysis. The cells were washed with PBS and processed for western blot analysis, as described previously (8). Antibodies against cleaved caspase-3 (Cell Signaling Technology Inc., Beverly, MD, USA), poly(ADP-ribose) polymerase (PARP) (Cell Signaling Technology Inc.) and glyceraldehyde 3-phosphate dehydrogenase (GAPDH; Trevigen, Gaithersburg, MD, USA) were used as primary antibodies. As secondary antibodies, we used $\alpha$ rabbit IgG (DAKO Japan) antibodies which were conjugated with horseradish peroxidase.

Fine cell structure. Near confluent HOK cells were inoculated for 0 or $24 \mathrm{~h}$ with $10 \mu \mathrm{M}$ doxorubicin. Cells detached by the cytotoxic 

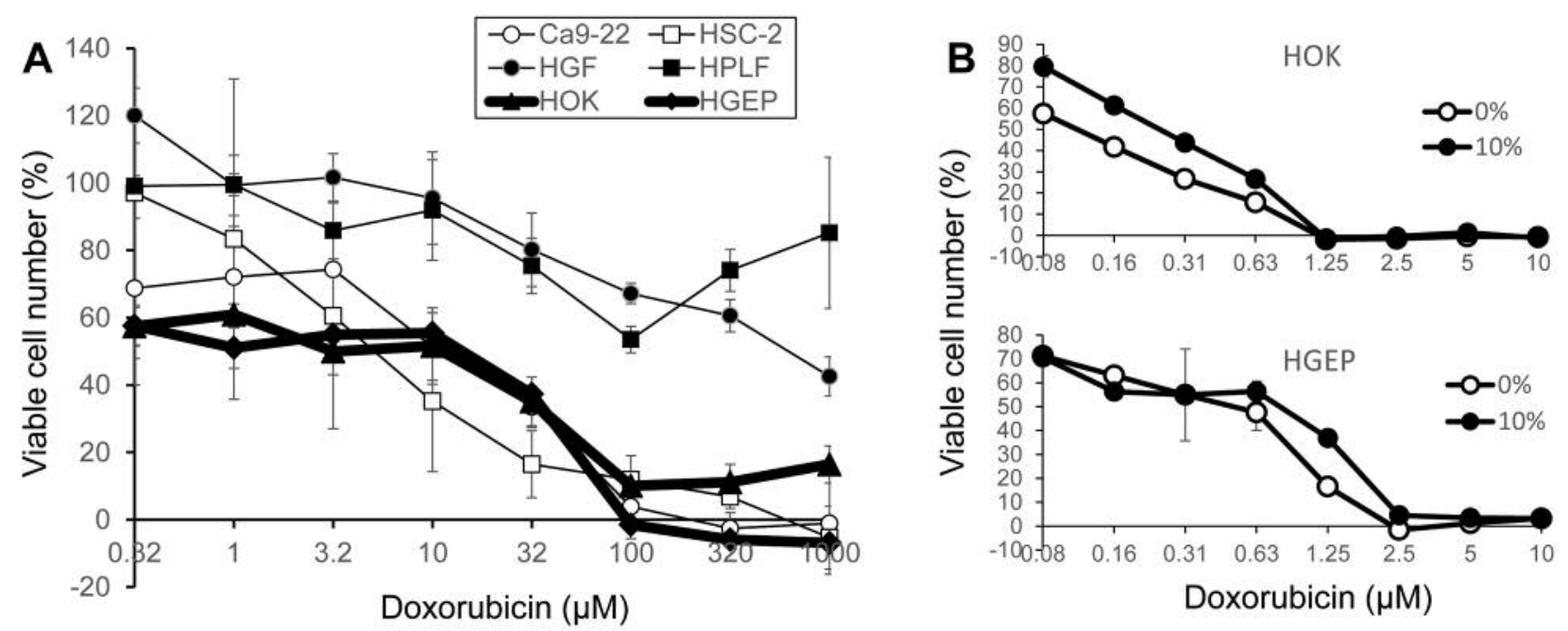

Figure 1. Doxorubicin induced potent cytotoxicity in human normal oral keratinocytes. (A) Comparison of doxorubicin sensitivity between human oral squamous cell carcinoma (Ca9-22, HSC-2), human normal oral mesenchymal (HGF, HPLF) and epithelial (HOK, HGEP) cells. Addition of as little as $0.32 \mu \mathrm{M}$ doxorubicin reduced the viability of both HOK and HPEP to approximately $60 \%$ of control. The viability declined with increasing concentrations of doxorubicin. Doxorubicin at all concentrations significantly reduced the viability $(p<005)$. (B) Effect of FBS concentration (either 0 or 10\%) on the doxorubicin sensitivity. HOK (upper panel) and HGEP (lower panel) cells were treated for $48 \mathrm{~h}$ with the indicated concentrations of doxorubicin in specified culture medium in the presence (-) or absence (O) of $10 \%$ FBS and the viable cell number was determined by MTT method, and expressed as percent of control (with the same amount of DMSO). Each value represents mean \pm S.D. values of triplicate determinations. Addition of as little as $0.08 \mu \mathrm{M}$ doxorubicin reduced the viability of both HOK and HPEP to 60 80\% of control. The viability declined with increasing concentrations of doxorubicin. Doxorubicin at all concentrations significantly reduced the viability $(p<005)$.

action of doxorubicin was recovered by centrifugation. Both adherent and detached cells were then fixed and processed for the observation under the JEM-1210 transmission electron microscope (JEOL, Co., Ltd., Akishima, Tokyo, Japan) (magnification: $\times 5000)$ as described previously (4).

Tumor-selectivity index calculation. The tumor-selectivity index (TS) was calculated by the following equation: $\mathrm{TS}=$ mean $\mathrm{CC}_{50}$ against normal cells/mean $\mathrm{CC}_{50}$ against tumor cells, that is, $\mathrm{B} / \mathrm{A}$ [HGF+HPLF+HPC (normal mesenchymal) (B) $v s$. Ca9-22+HSC2+HSC-3+HSC-4 (tumor epithelial) (A)], and C/A [HOK+HGEP (normal epithelial) (C) vs. Ca9-22+HSC-2+HSC-3+HSC-4 (tumor epithelial) (A)] (Table I). A, B and C are indicated in Table I.

Statistical treatment. Experimental data are the mean \pm standard deviation (SD). The statistical differences between control and treated groups were evaluated by Student's $t$-test. A value of $p<0.05$ was considered to be significant.

\section{Results}

Induction of keratinocyte toxicity by doxorubicin. Doxorubicin (DXR) showed higher cytotoxicity against two human normal oral keratinocytes (HOK, HGEP), as well as two human OSCC cell lines (Ca9-22, HSC-2), compared to two human normal oral mesenchymal cells (HGF, HPLF) (Figure 1A).

There was a possibility that higher sensitivity of human oral keratinocytes may be simply due to the absence of FBS, which might have produced higher amounts of unbound doxorubicin. This possibility, however, was eliminated by our finding that the addition of FBS (final 10\%) to HOK (upper panel) or HGEP (lower panel) cell culture medium only slightly reduced the cytotoxicity of doxorubicin (Figure 1B).

There was another possibility that higher sensitivity of human oral keratinocytes to doxorubicin may be due to its lower cell density, which may have keratinocytes exposed to higher concentrations of doxorubicin per a given number of cells. To test this possibility, OSCC cell lines (Ca9-22, HSC2 ), normal oral epithelial (HOK) and mesenchymal (HGF, HPLF) cells were inoculated at very low (LL), low (L), high $(\mathrm{H})$ or very high $(\mathrm{HH})$ cell density, and then treated with increasing concentrations of doxorubicin (Figure 2A-E). When the $\mathrm{CC}_{50}$ values thus obtained were plotted as a function of cell density (absorbance at $540 \mathrm{~nm}$ ), three different groups of cell populations were separated with each other (Figure 2F). Both keratinocytes and OSCC cell lines were more sensitive to mesenchymal cells, regardless of the cell density, eliminating this possibility.

Induction of apoptosis in keratinocyte by doxorubicin. When HOK cells were incubated for $24 \mathrm{~h}$ with $10 \mu \mathrm{M}$ doxorubicin, some part of the cell monolayer was detached from the culture dish. Observation under TEM demonstrated that the detached cells showed apoptotic morphology (i.e. loss of cell surface 

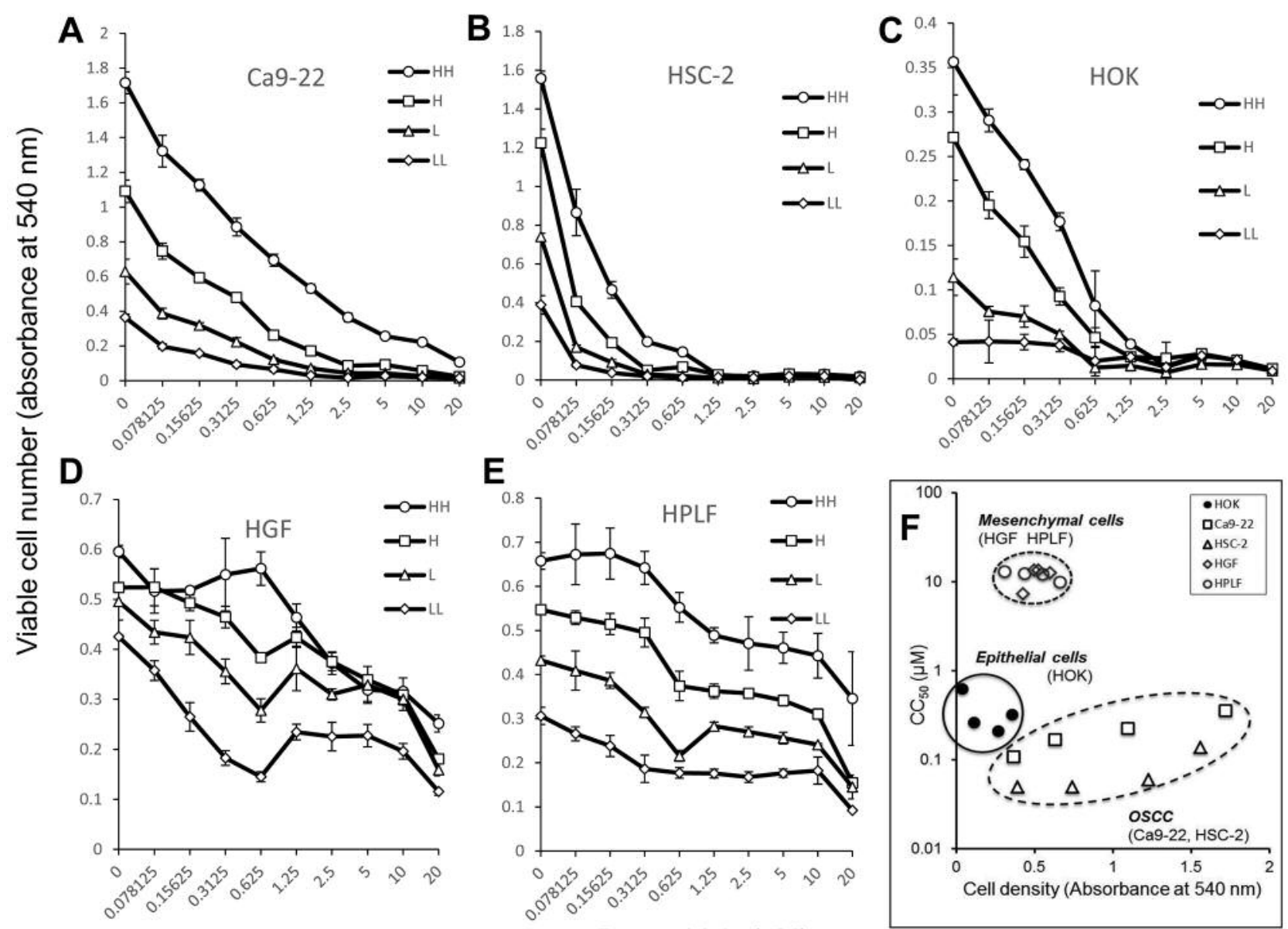

E
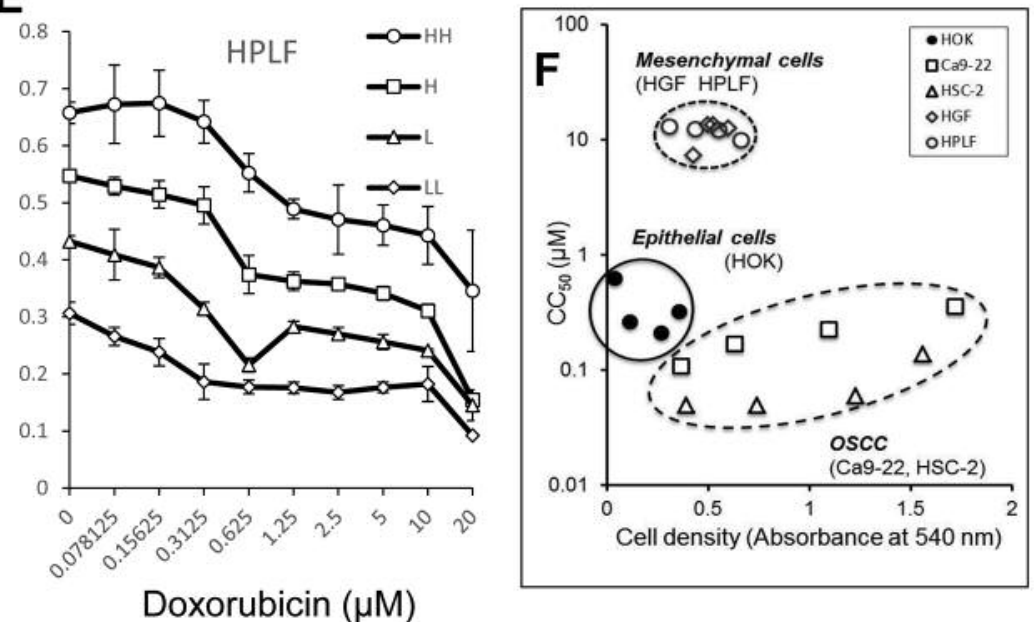

Figure 2. Keratinocyte toxicity induced by doxorubicin did not depend on cell density. Adherent Ca9-22 (A), HSC-2 (B), HOK (C), HGF (D) and $H P L F(E)$ cells were inoculated at very low $(L L)$, low $(L)$, high $(H)$ or very high $(H H)$ cell density, incubated for $48 h$ with the indicated concentrations of doxorubicin, and then the relative viable cell number (absorbance at $540 \mathrm{~m}$ ) was determined by the MTT method to calculate the $50 \%$ cytotoxic concentration $\left(C C_{50}\right)$. Each value represents mean $\pm S . D$. of triplicate determinations. Insert $(F): C C_{50}$ was plotted as a function of cell density (absorbance at $540 \mathrm{~nm}$ ) of control cells at the time of cell harvest.

microvilli, chromatin condensation, nuclear fragmentation) while attached cells preserved the autophagic morphology (i.e., multivascular body and lamella body) (Figure 3A). Western blot analysis demonstrated that treatment of HOK cells for $24 \mathrm{~h}$ with doxorubicin $(2 \mu \mathrm{M})$ induced the cleavage of PARP and caspase-3, suggesting the activation of caspase3 (Figure 3B). These data strongly suggest that keratinocyte toxicity by doxorubicin is coupled with induction of apoptosis.

Induction of keratinocyte toxicity by many anticancer drugs. We next investigated whether the keratinocyte toxicity is specific to doxorubicin, or a general phenomenon observed in all of anticancer drugs. To discriminate these possibilities, four epithelial OSCC cell lines (CA9-22, HSC-2, HSC-3, HSC-4), three normal mesenchymal cells (HGF, HPLF, HPC) and normal epithelial keratinocytes (HOK, HGEP) were treated with various concentrations of topoisomerase I inhibitors (camptothecin, SN-38, an active metabolite of irinotecan), topoisomerase II inhibitor (etoposide), antitumor antibiotics (doxorubicin, daunorubicin, mitomycin C), antimetabolites (methotrexate, 5-fluorouracil), antimicrotubule (docetaxel), alkylating agent (melphalan) or tyrosine kinase inhibitor (gefitinib) to determine the $\mathrm{CC}_{50}$ values. All these anticancer drugs showed excellent tumor-specificity (B/A) $(\mathrm{TS}=4$ 2708) when normal mesenchymal cells (B) were compared to epithelial OSCC cells (A). On the other hand, they showed much lower tumor-specificity (C/A) $(\mathrm{TS}=0.1 \sim 212)$ when normal keratinocytes (C) were compared to epithelial OSCC cells (A) (Table I). These data demonstrate that all 11 anticancer drugs showed potent keratinocyte toxicity. 
A

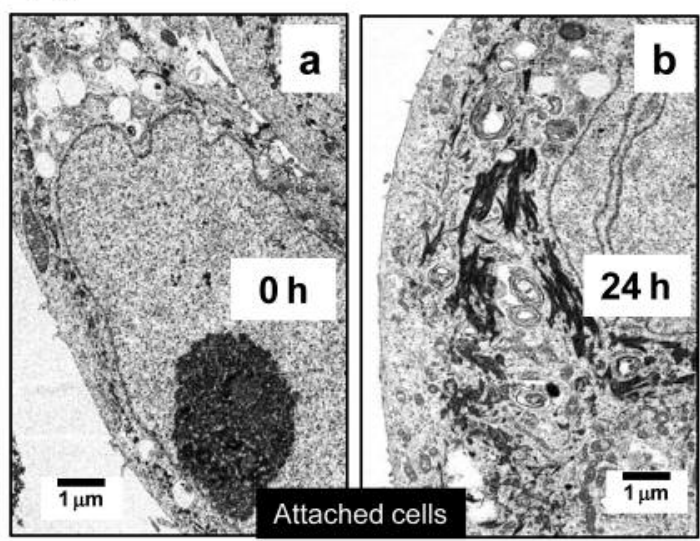

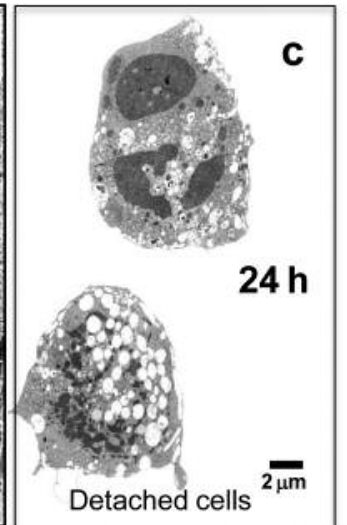

B

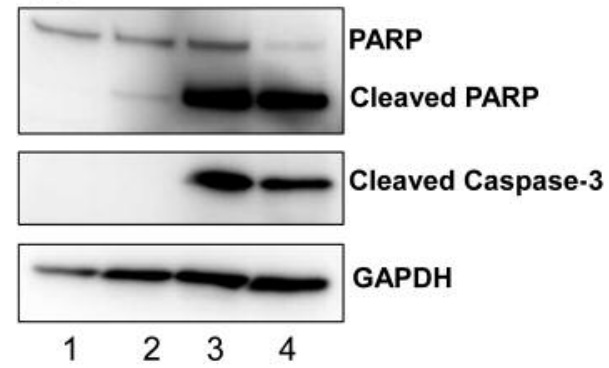

1. DMSO $0.2 \%$

2. Doxorubicin $0.4 \mu \mathrm{M}$

3. Doxorubicin $2 \mu \mathrm{M}$

4. Actinomycin D $1 \mu \mathrm{M}$

Figure 3. Doxorubicin induced apoptosis in HOK human normal oral keratinocyte. (A) Transmission electron microscope (TEM) analysis. HOK cells were treated for $O(a)$ or $24(b, c) h$ with $10 \mu M$ doxorubicin and fine cell structure of adherent $(a, b)$ and detached cells $(c)$ were observed under TEM. Bars indicate $1 \mu \mathrm{m}(a, b)$ and $2 \mu \mathrm{m}(c)$. (B) Western blot analysis. HOK cells were treated for $24 \mathrm{~h}$ with either vehicle (DMSO) (0.2\%) (lane 1), doxorubicin $(0.4$ and $2 \mu \mathrm{M})$ (lane 2 and 3) or actinomycin D (1 $\mu \mathrm{M})$ (lane 4). Detached and attached cells were combined for western blot analysis.

\section{Discussion}

The present system demonstrated that doxorubicin induced very potent toxicity against human oral keratinocytes, regardless of culture conditions (presence or absence of FBS, cell density), accompanied by apoptosis induction (Figure 3). It should be noted that during detachment from the culture plate, cells were committed to apoptosis (Figure 3A-C) while attached cells still preserved the autophagic phenotype (Figure 3A-B). We have previously reported that (E)-3-(4-hydroxystyryl)-6-methoxy-4H-chromen-4-one, having high tumor-specificity $(\mathrm{TS}=69)$ and lower keratinocyte toxicity, induced apoptosis in HSC-2 cells after autophagy was suppressed (4). These data suggest that suppression of autophagy triggers apoptosis induction, in accordance with the generally accepted role of autophagy in maintaining the long-term survival of disseminated residual cells $(9,10)$.

The present study demonstrated that doxorubicin induced apoptosis also in human normal oral keratinocytes, like that has previously been published in various cancer cells (1113). This indicates the induction of apoptosis itself does not guarantee the anti-tumor potential. A simple search in pubmed with two key words, flavonoids and apoptosis, got over nearly 8000 publications, even though most of flavonoids showed lower levels of tumor-specificity (TS values=1.3 3.2) (3). Taken together, tumor-specificity, rather than apoptosis-inducing activity, should be used for the evaluation of antitumor activity.

We found that most of anticancer drugs showed keratinocyte toxicity (Table I). This points out that keratinocyte toxicity is new adverse effect of anticancer agents, in addition to cardiomyopathy (11). Further study is required to elucidate why human oral keratinocytes are more sensitive to anticancer drugs compared to human mesenchymal cells.

It, thus, became very important to search for natural products that can alleviate the doxorubicin-induced keratinocyte toxicity. We found that alkaline extract of the leaves of Sasa senanensis Rehder (SE), OTC drug, having prominent antiviral (14) and anti-UV activity (15), significantly alleviated the DXR-induced cytotoxicity against HGEP, by its growth-promoting activity (Figure 4A). On the other hand, the growth promoting effect of SE against OSCC cell lines, Ca9-22) (B), HSC-2 (C) and HSC-3 (D) was significantly less (Figure 4). Further study is required to identify the active ingredient of SE that promote the growth of HGEP.

In conclusion, the present paper suggests that keratinocyte toxicity may be the new adverse effect of most of anticancer agents.

\section{Conflicts of Interest}

The first Author (HS) was supported by the Daiwa Biological Research Institute Co., Ltd., Kanagawa, Japan. The authors wish to confirm that such financial support has not influenced the outcome or the experimental data.

\section{Acknowledgements}

This work was partially supported by KAKENHI from the Japan Society for the Promotion of Science (JSPS) (16K11519). 


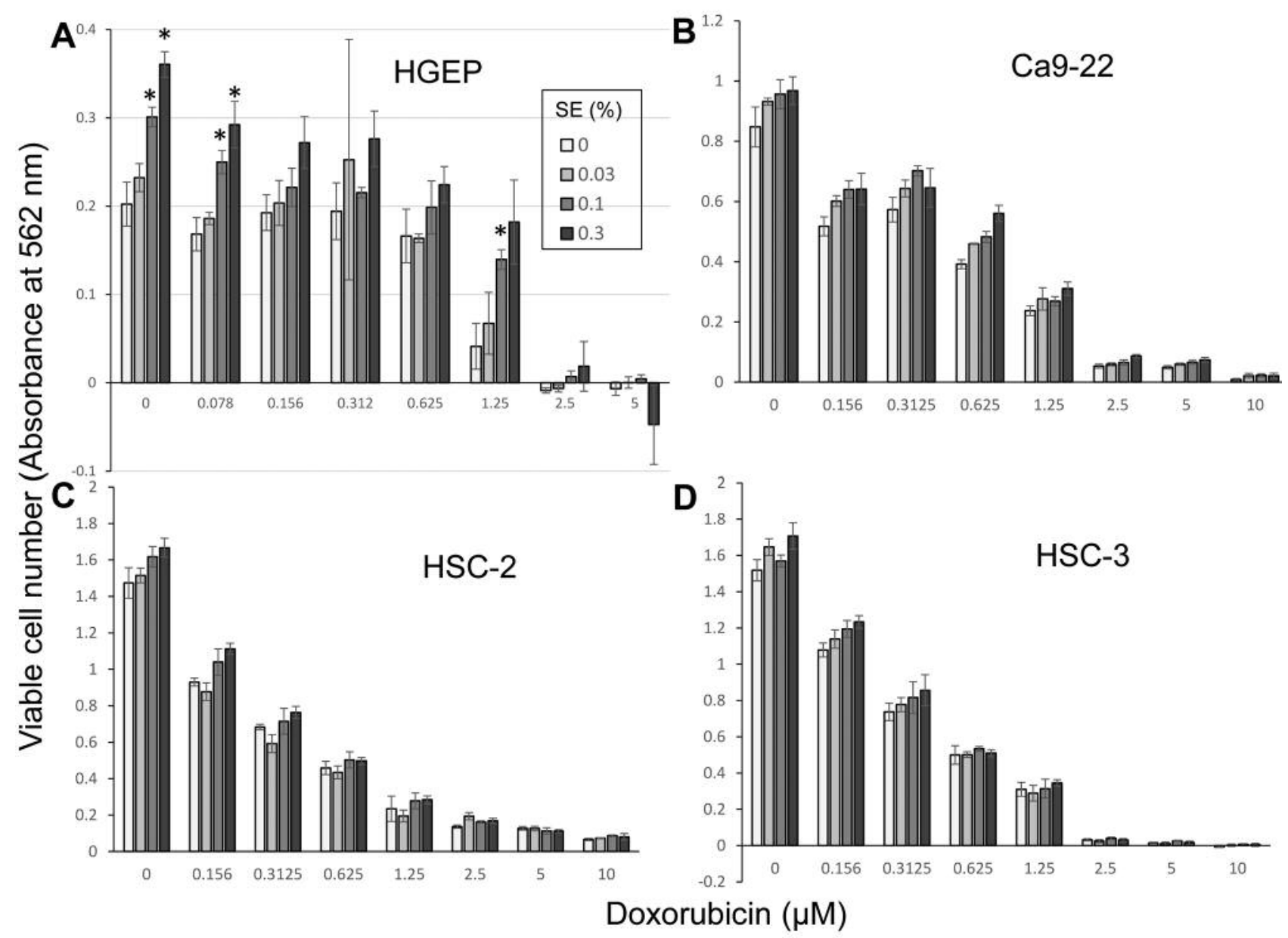

Figure 4. Protective effect of alkaline extract from the leaves of Sasa senanensis Rehder (SE) on doxorubicin cytotoxicity. HGEP (A), Ca9-22 (B), $H S C-2(C)$ or HSC-3 (D) cells were incubated for $48 \mathrm{~h}$ with the indicated concentrations of doxorubicin in the presence of 0, $0.03,0.1$ or $0.3 \%$ SE, and the viable cell number (absorbance at $562 \mathrm{~mm}$ ) was determined by the MTT method. Each value represents mean $\pm S . D$. of triplicate determinations. Asterisks indicate statistical significance at $p<0.05$.

\section{References}

1 Emoto C, Yoda N, Uno Y, Iwasaki K, Umehara K, Kashiyama $\mathrm{E}$ and Yamazaki H: Comparison of P450 enzymes between cynomolgus monkeys and humans: P450 identities, protein contents, kinetic parameters, and potential for inhibitory profiles. Curr Drug Metab 14(2): 239-252, 2013.

2 Cao X, Gibbs ST, Fang L, Miller HA, Landowski CP, Shin HC, Lennernas H, Zhong Y, Amidon GL, Yu LX and Sun D: Why is it challenging to predict intestinal drug absorption and oral bioavailability in human using rat model. Pharm Res 23(8): 1675-1686, 2006.

3 Sakagami H: Biological activities and possible dental application of three major groups of polyphenols. J Pharmacol Sci 126: 92106, 2014

4 Sakagami H, Shimada C, Kanda Y, Amano O, Sugimoto M, Ota S, Soga T, Tomita M, Sato A, Tanuma S, Takao K and Sugita Y: Effects of 3-styrylchromones on metabolic profiles and cell death in oral squamous cell carcinoma cells. Toxocology Reports 2: 1281-1290, 2015.
5 Sakagami H, Uesawa Y, Ishihara M, Kagaya H, Kanamoto T, Terakubo S, Nakashima H, Takao K and Sugita Y: Quantitative structure-cytotoxicity relationship of oleoylamides. Anticancer Res 35: 5341-5355, 2015.

6 Uesawa Y, Sakagami H, Kagaya H, Yamashita M, Takao K and Sugita Y: Quantitative structure-cytotoxicity relationship of 3benzylidenechromanones. Anticancer Res 36(11): 5803-5812, 2016.

7 Kantoh K, Ono M, Nakamura Y, Nakamura Y, Hashimoto K, Sakagami $\mathrm{H}$ and Wakabayashi $\mathrm{H}$ : Hormetic and anti-radiation effects of tropolone-related compounds. In Vivo 24: 843-852, 2010.

8 Suzuki R, Matsushima Y, Okudaira N, Sakagami H and Shirataki Y: Cytotoxic components against human oral squamous cell carcinoma isolated from Andrographs paniculata. Anticancer Res 36(11): 5931-5935, 2016.

9 Adhauliya N, Kalappanavar AN, Ali IM and Annigeri RG: Autophagy: A boon or bane in oral cancer. Review. Oral Oncol 61: 120-126, 2016.

10 Sosa MS, Bragado P, Debnath J and Aguirre-Ghiso JA: Regulation of tumor cell dormancy by tissue microenvironments and autophagy. Review. Adv Exp Med Biol 734: 73-89, 2013. 
11 Kankeu C, Clarke K, Passante E and Huber HJ: Doxorubicininduced chronic dilated cardiomyopathy-the apoptosis hypothesis revisited. Review. J Mol Med (Berl), 2016. doi: 10.1007/s00109-016-1494-0. [Epub ahead of print]

12 Tacar O and Dass CR: Doxorubicin-induced death in tumour cells and cardiomyocytes: is autophagy the key to improving future clinical outcomes? Review. J Pharm Pharmacol 65(11): 1577-1589, 2013

13 Suzuki F, Hashimoto K, Kikuchi H, Nishikawa H, Matsumoto H, Shimada J, Kawase M, Sunaga K, Tsuda T, Satoh K and Sakagami H: Induction of tumor-specific cytotoxicity and apoptosis by doxorubicin. Anticancer Res 25(2A): 887-893, 2005
14 Sakagami H, Fukuchi K, Kanamoto T, Terakubo S, Nakashima $\mathrm{H}$, Natori T, Suguro-Kitajima M, Oizumi H, Yasui T and Oizumi T: Synergism of alkaline extract of the leaves of Sasa senanensis Rehder and antiviral agents. In Vivo 30(4): 421-426, 2016.

15 Sakagami H, Sheng H, Okudaira N, Yasui T, Wakabayashi H, Jia J, Natori T, Suguro-Kitajima M, Oizumi H and Oizumi T: Prominent anti-UV activity and possible cosmetic potential of lignin-carbohydrate complex. Review. In Vivo 30(4): 331-339, 2016.

Received December 14, 2016

Revised January 30, 2017

Accepted January 31, 2017 